\title{
VORTEX EQUILIBRIA IN FLOW PAST A PLATE
}

\author{
N. ROBB MCDONALD ${ }^{1}$
}

(Received 10 August, 2007; revised 14 November, 2007)

\begin{abstract}
Families of vortex equilibria, with constant vorticity, in steady flow past a flat plate are computed numerically. An equilibrium configuration, which can be thought of as a desingularized point vortex, involves a single symmetric vortex patch located wholly on one side of the plate. Given that the outermost edge of the vortex is unit distance from the plate, the equilibria depend on three parameters: the length of the plate, circulation about the plate, and the distance of the innermost edge of the vortex from the plate. Families in which there is zero circulation about the plate and for which the Kutta condition at the plate ends is satisfied are both considered. Properties such as vortex area, lift and free-stream speed are computed. Time-dependent numerical simulations are used to investigate the stability of the computed steady solutions.
\end{abstract}

2000 Mathematics subject classification: 76B47, 76B10.

Keywords and phrases: vortex dynamics, vortex equilibria, contour dynamics, aerodynamics.

\section{Introduction}

The formation of closed streamlines is a characteristic feature of two-dimensional flow past a body. This observation has led to a number of studies of trapped vortices in steady flow of an inviscid fluid past a body, that is, vortex equilibria. For example, in uniform flow past a circular cylinder, the Föppl vortices (see for example [13]) are a family of standing point vortex pairs, of equal and opposite circulation, arranged symmetrically downstream of the cylinder. Elcrat et al. [4] showed numerically that the Föppl equilibria can be desingularized into a family of increasing-area constantvorticity vortex patches with the same circulation as the Föppl point vortices. The constant-vorticity assumption is appropriate in this scenario since it is consistent with the Prandtl-Batchelor theorem [1], which asserts that vorticity within closed streamlines becomes uniform in the limit of zero viscosity. In addition to vortex equilibria behind the cylinder, Elcrat et al. [4] also found a family of vortex patch

\footnotetext{
${ }^{1}$ Department of Mathematics, University College London, London WC1E 6BT, UK; e-mail: robb@math.ucl.ac.uk.

(C) Australian Mathematical Society 2008, Serial-fee code 0334-2700/08
} 
equilibria located directly above and below the cylinder. These were later shown to be unstable [5]. Recently, Hocking [6] has used boundary integral methods to find a family of solutions describing a constant-vorticity symmetric wake (a vortex pair) attached to a circular cylinder and extending downstream a finite distance.

In this work the body considered is a flat plate of finite length aligned with the flow, and equilibria comprising only one vortex located on one side of the plate are investigated. The vortex boundary is assumed symmetric in the flow direction. The asymmetry in the direction perpendicular to the plate permits steady flows with an arbitrary circulation around the plate. This, in turn, implies that, in general, there will be a lift on the plate. Two cases are considered here: (i) zero circulation around the plate and (ii) when the circulation is chosen such that the Kutta condition at the ends of the plate is satisfied, that is, the velocity at the plate ends is bounded. Case (i) may arise, for example, in a situation in which a vortex initially distant from the plate is advected towards the plate and is subsequently trapped. The circulation about the plate, which is initially zero, remains so by Kelvin's circulation theorem. Case (ii) generalizes that of Saffman [13] who considered the case of a single point vortex located above the midpoint of a flat plate aligned parallel to the flow in which the Kutta condition is satisfied. Saffman's solution is given in Section 2.2 since it represents the singular limit of the more general family of vortex patch solutions found here. With aerodynamic applications in mind Saffman and Sheffield [14] also considered the case of point vortices in equilibrium with a plate at incidence, and Saffman and Tanveer [15] numerically calculated uniform vortices bounded by a vortex sheet attached to a plate at incidence with a forward-facing flap. These studies showed that the presence of the vortex can enhance the lift on the plate.

A practical use of the present work is that comparison of results for the more realistic vortex patches obtained here with those for point vortices will enable quantitative judgment of the ability of the simpler point vortex models to predict properties such as lift in, say, aerodynamic applications.

The equilibria found here may also be interpreted as generalizations of the family of uniform vortex patches in steady translation near an infinitely long plane wall. Such vortices, also known as V-states, have attracted substantial interest in the vortex dynamics literature - see for example $[2,11,16]$. In fact the numerical algorithm used here to find steady solutions is partly based on that due to Pierrehumbert [11]. In a uniform flow with speed equal and opposite to their translational velocity, V-states are stationary and the equilibria found here should tend to these previous solutions in the limit of large plate length.

In Section 2 properties of families of point vortex equilibria are derived from the Hamiltonian for uniform flow past a finite plate in the presence of a point vortex. The existence of such equilibria provides the motivation for the numerical construction of analogous desingularized vortex patch equilibria. The numerical method for constructing the steady solutions, based on contour dynamics, is described in Section 3. Results including vortex shapes, area, upstream flow speed, and lift on the plate are given in Section 4. In Section 5 the robustness of the computed 


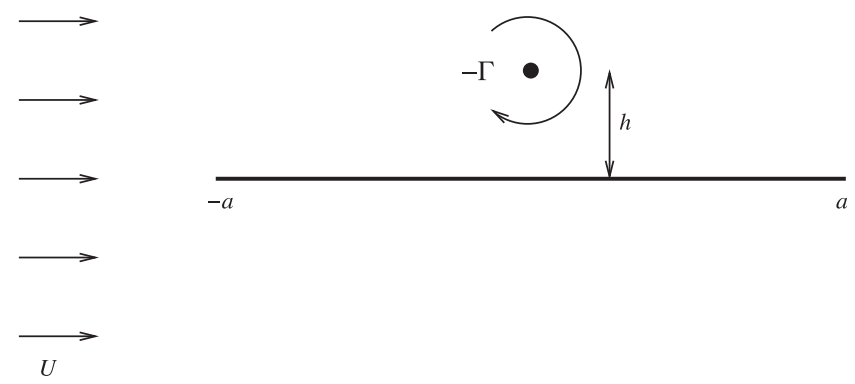

FIGURE 1. Point vortex equilibrium for flow past a plate. Note that, if $U>0$, then the vortex circulation must be negative.

equilibria is tested by using them as initial conditions in a time-dependent contour dynamics simulation.

\section{Point vortex equilibria near a flat plate}

Consider a point vortex of circulation $\Gamma$ in a uniform stream with velocity $U$ parallel to a flat plate of length $2 a$-see Figure 1 . The fluid is inviscid and the flow is steady. Let $z=x+i y$ be a complex coordinate system such that $\operatorname{Im} z=0$ is coincident with the plate and $z=0$ is its midpoint.

In this section the vortex Hamiltonian is used to establish equilibrium conditions in terms of the parameters $h, \Gamma, a$ and $U$. Alternatively, these conditions could be found from the complex potential and requiring that the non-self-induced velocity at the vortex vanishes. However, the vortex Hamiltonian, in which level curves of the Hamiltonian coincide with vortex trajectories (see for example [13]), has the advantage that it also gives information on the stability properties of the equilibria (see for example [17]).

Let the vortex Hamiltonian in the $z$-plane be $H_{z}$. The velocity of the vortex is obtained from the relations $u=\partial H_{z} / \partial y, v=-\partial H_{z} / \partial x$. One can construct $H_{z}$ by first noting that the Hamiltonian for a point vortex of circulation $\Gamma$ outside the unit circle in the $\zeta$-plane is (see for example [17])

$$
H_{\zeta}=-\frac{\gamma}{4 \pi} \log |\zeta|^{2}+\frac{\Gamma}{4 \pi} \log \left(|\zeta|^{2}-1\right)
$$

where $\gamma$ is the (arbitrary) strength of a point vortex at the centre of the circle. Using Routh's rule $[12,13]$ the Hamiltonian in the $z$-plane is

$$
H_{z}=H_{\zeta}+\frac{\Gamma}{4 \pi} \log \left|\frac{d z}{d \zeta}\right|,
$$

where $z=(a / 2)\left(\zeta+\zeta^{-1}\right)$ maps the unit circle in the $\zeta$-plane to a flat plate of length $2 a$ in the $z$-plane. Including a uniform stream with velocity $U,(2.1)$ 
and (2.2) give

$$
H_{z}=U y+\frac{\Gamma}{4 \pi} \log \left(|\zeta|^{2}-1\right)-\frac{\gamma}{2 \pi} \log |\zeta|+\frac{\Gamma}{4 \pi} \log \left|1-\zeta^{-2}\right|,
$$

where $\zeta=\zeta(z)$. Note that $\gamma-\Gamma$ is the net circulation about the plate.

Let the vortex be at $z=i h$, which in the $\zeta$-plane corresponds to

$$
\zeta=i\left(h+\sqrt{h^{2}+a^{2}}\right) / a=i b,
$$

where $b>1$. At the vortex $v=0$ by symmetry and $H_{z}=H_{z}(0, b)$. The condition that the vortex be stationary with respect to the plate is then $\partial H_{z}(0, b) / \partial b=0$. This gives a relation between $U, a, h, \Gamma$ and $\gamma$ for stationarity:

$$
-\frac{U a}{2}\left(1+b^{-2}\right)=\frac{\Gamma}{\pi} \frac{b^{3}}{b^{4}-1}-\frac{\gamma+\Gamma}{2 \pi b},
$$

where $b=b(h)=\left(h+\sqrt{h^{2}+a^{2}}\right) / a$.

2.1. Zero circulation about the plate In this case $\gamma=\Gamma$ and (2.4) reduces to

$$
\frac{\Gamma}{4 \pi U}=-\frac{h\left(1+b^{2}\right)^{2}}{4} .
$$

Note that, if $h, U>0$, then $\Gamma<0$ for the vortex to be stationary.

The lift on the plate is, in general, given by $L=U \gamma$ (see [10,13]) and for this case is

$$
L=-\frac{\Gamma^{2}}{\pi h\left(1+b^{2}\right)^{2}} .
$$

Note that, if $h>0$, then $L<0$, that is, the lift is in the negative $y$-direction.

2.2. Kutta condition This case is also discussed in Saffman [13]. The Kutta condition is satisfied when the velocity at the ends of the plate is finite. Let $w$ be the complex potential. Since $u-i v=d w / d z=(d w / d \zeta)(d \zeta / d z)$ and $d \zeta / d z$ is unbounded at the plate ends, this is equivalent to demanding that $d w /\left.d \zeta\right|_{\zeta= \pm 1}=0$. Using the velocity potential for a point vortex outside the unit $\zeta$ cylinder (the uniform stream is irrelevant here since $\zeta= \pm 1$ correspond to stagnation points in uniform flow past a cylinder), the complex potential is

$$
w=-\frac{i \Gamma}{2 \pi} \log \left(\frac{\zeta-i b}{\zeta-i b^{-1}}\right)-\frac{i \gamma}{2 \pi} \log \zeta
$$

and the Kutta condition is satisfied when (see also [13, Page 122, Equation (18)])

$$
\frac{\gamma}{\Gamma}=\frac{b^{2}-1}{b^{2}+1} \text {. }
$$

Using (2.8) in (2.4) gives

$$
\frac{\Gamma}{4 \pi U}=-\frac{h\left(1+b^{2}\right)^{2}}{4 b^{2}}
$$



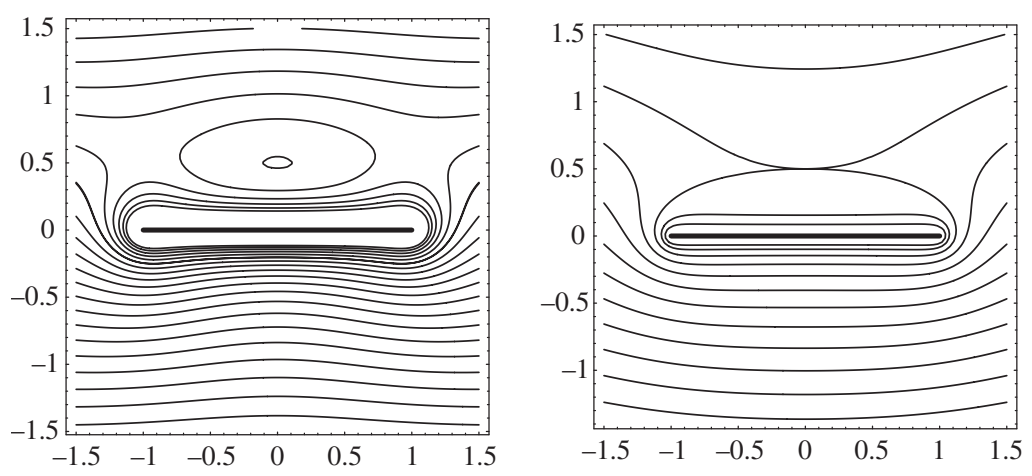

FIGURE 2. Contours of the Hamiltonian for zero circulation about the plate (left) and when the Kutta condition is satisfied (right) with $U=0.1$ and $h=0.5$.

with associated lift on the plate

$$
L=-\frac{2 \Gamma^{2}}{\pi a}\left(\frac{b}{b^{2}+1}\right)^{3} .
$$

As in the zero circulation case $h, U>0$ implies $\Gamma, L<0$.

In the limit of large plate length $a \rightarrow \infty$ and fixed $h$ it follows that $b \rightarrow 1$ and, from (2.5) and (2.9), the ratio $\Gamma / 4 \pi U \rightarrow-h$. This is precisely the condition for a vortex of circulation $\Gamma$ and distance $h$ from an infinitely long plane wall to remain stationary in a uniform stream with velocity $U$.

Contours of the Hamiltonians for the two cases of zero circulation and Kutta flow are given in Figure 2. In both cases $h=0.5$ and $U=0.1$. In addition, $\Gamma$ and (in the case of Kutta flow) $\gamma$ are chosen such that (2.5) or (2.9) are satisfied respectively. Since contours of the Hamiltonian correspond to trajectories of the point vortex, it is clear from Figure 2, as expected from the choice of parameters, that $z=0.5 i$ corresponds to an equilibrium point. The contours in the zero circulation case indicate the presence of further equilibrium points either side of the equilibrium point centred above the plate, but these are not investigated here. Interestingly, the nature of the equilibrium point is different in the two cases, being elliptic in the zero circulation case and hyperbolic in the Kutta case. This suggests different stability properties between the equilibria, namely that the zero circulation equilibria are stable and those satisfying the Kutta condition are unstable (see for example [17]). In principle it is possible to derive an explicit condition determining the elliptic or hyperbolic nature of the critical point by examining the determinant of the Hessian of the Hamiltonian near the critical point. However, this proves to be an awkward calculation and is not pursued here. It is noteworthy, however, that the behaviour shown in Figure 2 appears to be generic: many examples, covering a wide range of parameter space of the Hamiltonian were plotted and all showed elliptic equilibrium points directly above the plate in the zero circulation case and hyperbolic points in the Kutta case. The stability question is 


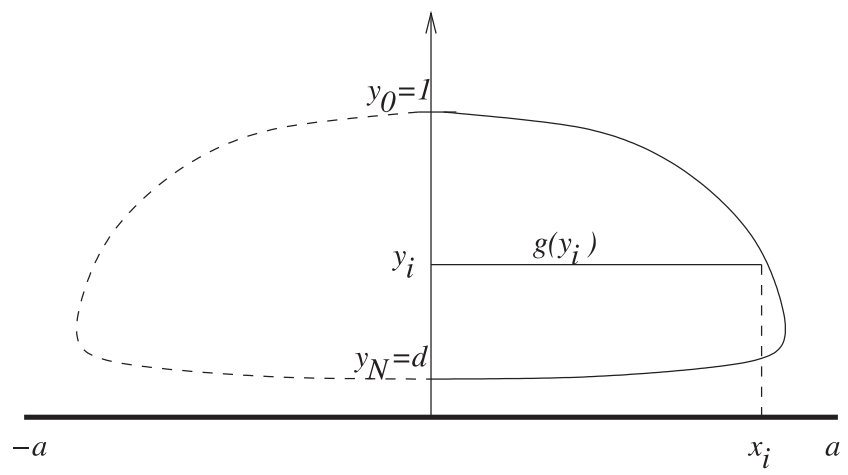

FIgURE 3. Vortex patch above plate of length $2 a$. The patch is symmetric about the $y$-axis. In the numerical computations $y_{0}=1$ is fixed and $y_{N}=d$ is a parameter.

returned to in Section 5 when the vortex patch equilibria are tested in time-dependent simulations.

\section{Numerical method}

Consider a symmetric vortex patch of uniform vorticity $\omega$ centred over a plate of width $2 a$. Using the normal distance of the outermost edge of the patch from the wall as the lengthscale and $|\omega|^{-1}$ as the timescale, the system is nondimensionalized. Hence the outermost edge of the patch is unit distance from the plate and its innermost edge is distance $d$ from the plate and the patch vorticity is -1 (see Figure 3). Since the patch has uniform vorticity, the numerical method of contour dynamics (a type of boundary integral method, see for example [3]) can be used for efficient and accurate computation of velocities due to the vortex patch alone. Indeed, the computation of steady flows with vorticity was a primary reason for the original development of contour dynamics $[2,16]$.

For an infinitely long wall, the computation of velocities induced by the patch can be calculated straightforwardly by adding in an image patch, see for example $[11,16]$. For a finite plate image-based methods are not possible and instead the method developed and used by Johnson and McDonald [7-9] for the study of vortex motion in the presence of boundaries (for example circular islands, gaps in walls, plates) is used. Briefly, in the present case, this can be done by first ignoring the presence of the plate and using a standard contour dynamics algorithm (see for example [3]) to compute the velocity field at nodes on the contour and at points along the plate. By mapping the exterior of the plate to the exterior of the unit circle in the $\zeta$-plane, the velocity data on the plate boundary are then used to compute a unique irrotational velocity field, with complex potential

$$
w_{b}=\sum_{n=1}^{\infty} c_{n} \zeta^{-n}
$$


which when added to the original velocity field satisfies the zero normal flow boundary condition on the plate. In practice this involves truncating the sum in (3.1) at $n=N$ and determining a finite number of complex coefficients $c_{n}$ using Fourier methods (see [7] for further details). The total velocity at the nodes of the patch is then the sum of those due to the patch alone and those due to the presence of the plate, which are given by

$$
u_{b}-i v_{b}=\frac{d w_{b}}{d z}=\frac{d w_{b}}{d \zeta} \frac{d \zeta}{d z}=-\frac{2}{a}\left(1-\zeta^{-2}\right)^{-1} \sum_{n=1}^{N} n c_{n} \zeta^{-n-1}
$$

Further, the circulation about the plate can be set to its required value by adding the term $-i \kappa /(2 \pi \zeta)$, where $\kappa$ is the circulation about the plate, to the complex velocity field. As in the point vortex case, the circulation about the plate can be expressed as $\kappa=\gamma-\Gamma$ where $\Gamma$ is now the circulation of the patch (that is, the product of vorticity and patch area) and $\gamma$ is an additional arbitrary circulation around the plate.

In the case of zero circulation about the plate the choice $\kappa=0$ is made. In the Kutta case, recall that it is required that the velocity at the plate ends be bounded, which, in turn, implies that the velocity in the $\zeta$-plane vanishes at $\zeta= \pm 1$. This is straightforward to implement numerically. The velocity computed at the ends of the plate due to the patch alone is necessarily finite, thus only the correction velocity $u_{b}-i v_{b}$ and $-i \kappa / 2 \pi \zeta$ in the $\zeta$-plane need be considered. Thus it is required that, at $\zeta= \pm 1$

$$
\frac{d w_{b}}{d \zeta}-\frac{i \kappa}{2 \pi \zeta}=0
$$

This gives $\kappa=-\left.2 \pi v_{b}\right|_{\zeta=1}$. As a check on this numerical procedure, the results in the Kutta case were tested by comparing the analytical point vortex solution discussed in Section 2 with a small patch such that $d=0.95$. Such a patch is very close to circular and hence induces a velocity field outside the patch which closely approximates that of a point vortex. Choosing the circulation of the point vortex equal to that of the small patch, agreement was found to three significant figures between numerically computed values of $U$ and $\gamma$ and those predicted explicitly by (2.8) and (2.9).

The patch boundary is discretized into nodes at $z_{i}=x_{i}+i y_{i}$. Initially the boundary is assumed circular with diameter $1-d$ and centre $(1+d) / 2$ and the nodes are uniformly spaced around the circle. Note that between nodes there is a nonuniform sine-type spacing in the $y$-direction which maintains accuracy near $y=d$ and $y=1$. For a given vortex shape, $d$, and plate length, $a$, velocities are computed on the vortex boundary using contour dynamics and (3.2), enabling the computation of the streamfunction $\psi_{P}\left(x_{i}, y_{i}\right)$ due to the plate and the patch itself. The iterative numerical method of [11] is then used. Briefly, this involves changing the shape of the vortex (with $z_{1}$ and $z_{N}$ fixed) and the free-stream velocity $U$ so that the streamfunction $\psi\left(x_{i}, y_{i}\right)=\psi_{P}\left(x_{i}, y_{i}\right)-U y_{i}$, where $U=\left(\psi_{P}(0,1)-\psi_{P}(0, d)\right) /(1-d)$, becomes constant along the vortex boundary. Velocities, and hence $\psi_{P}$, at nodal points are recomputed at each iteration. During this process $y_{i}$ is fixed and the distance $x_{i}=g\left(y_{i}\right)$ 


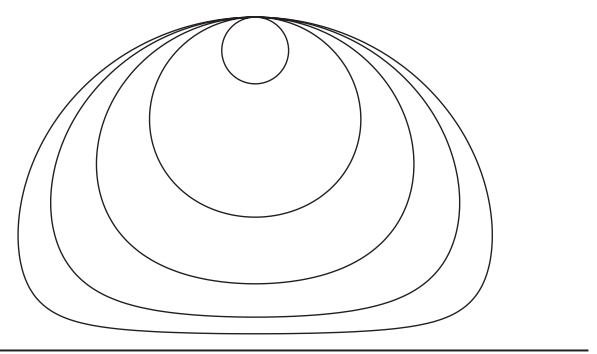

FIGURE 4. Vortex patch shapes for $d=0.05,0.1,0.2,0.4,0.8$ with zero circulation about the plate and $a=1$. The aspect ratio of the plot is one.

is adjusted. Once the streamfunction becomes constant on the boundary of the patch (to within some numerical tolerance) a steady solution is realized. The patch area, $U$ and circulation about the plate are computed at each iteration and are all monitored for convergence.

In the results reported here a resolution of 0.01 between adjacent nodes on the patch boundary was used, with a similar resolution used along the plate to calculate the irrotational field due to the presence of the plate. The sum in (3.2) was truncated at the integer closest to $N=50 a$, where $a$ is the half-length of the plate. In all results, convergence of solution properties such as vortex area and $U$ were obtained to four significant figures in typically 50 iterations.

\section{Results}

4.1. Zero circulation about the plate Figure 4 shows shapes of equilibrium vortex patches above a plate of length $a=1$ for $d=0.05,0.1,0.2,0.4$ and 0.8 . As $d$ increases the patch becomes more circular. Since the flow exterior to a circular vortex patch is the same as a point vortex with the same circulation, it is expected that for $d \geq 0.4$ the point vortex model provides a good approximation for the patch. The flattening of the vortex boundary as it approaches the plate is typical of the shape of $\mathrm{V}$-states near an infinitely long wall $[11,16]$.

Figure 5 shows the effect of increasing the plate length $a$ with fixed $d=0.05$. As $a$ increases, the area of the patch increases. Also shown is the limiting case $a=\infty$ corresponding to an infinitely long wall obtained using Pierrehumbert's original algorithm [11]. For $a \geq 4$ the infinitely long wall case provides a good approximation for the shape of the patch.

Properties of the equilibria for $d=0.05$ are plotted in Figure 6 as a function of $a$ (choosing $a=0.5,0.75,1,1.5,2,3,4,5,6,7,8,9,10)$. As $a$ increases so does the area of the equilibrium patch, rapidly approaching the area for the infinitely long wall case. The larger the patch the greater its circulation and hence the larger the velocity $U$ needs to be in order to maintain equilibrium as demonstrated in the second plot. The variation of the ratio $\Gamma / 4 \pi U$ as a function of $a$ shows good agreement with the theoretical result (2.5) for a point vortex with the choice $h=0.525$ (the mean 


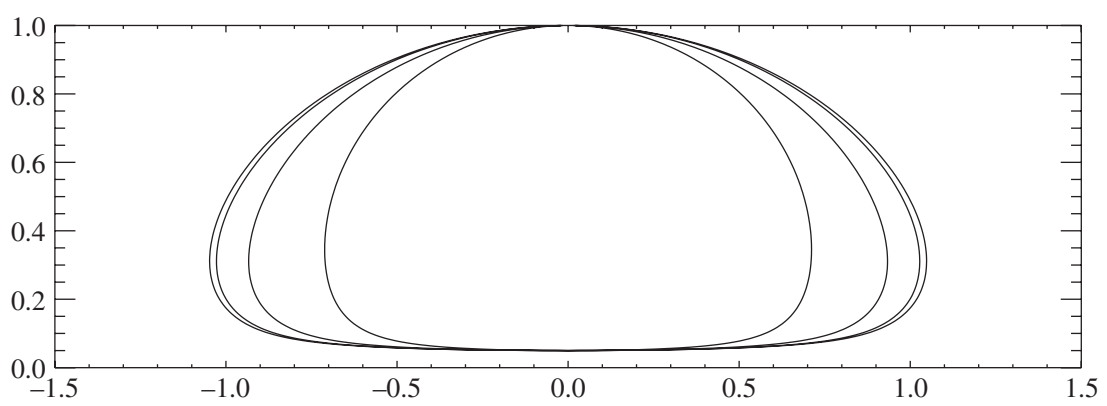

FIGURE 5. Vortex patch shapes of increasing area for $a=1,2,4, \infty$ with zero circulation about the plate and $d=0.05$.
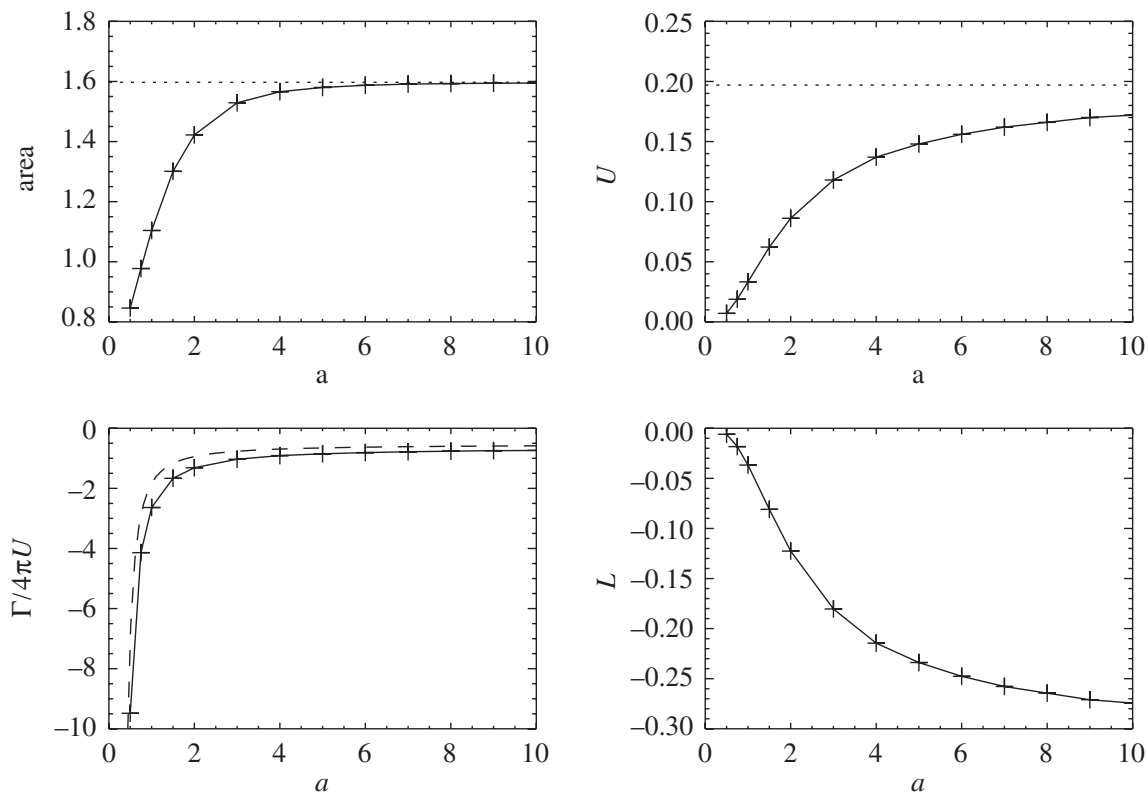

FIGURE 6. Equilibria properties of patch area, $U, \Gamma / 4 \pi U$ and lift $L$ for $d=0.05$ as a function of $a$. In the top two plots the dotted line represents patch area and $U$ for an infinitely long wall. In the third plot the dashed line represents the value of $\Gamma / 4 \pi U$ for a point vortex (2.5).

distance between 1 and $d$ ). That the agreement is reasonable for such a noncircular vortex implies that the agreement should be even better for larger values of $d$. This is indeed the case: a similar plot (not shown) for $d=0.8$ demonstrates the ratio $\Gamma / 4 \pi U$ as a function of $a$ for the patch and a point vortex with the same circulation are virtually identical. This again strongly suggests that the point vortex model is a good model for the patch for most values of $d$. Finally, note that the lift increases in magnitude with $a$. This is because of the increase in circulation of the patch as its area grows with $a$. 


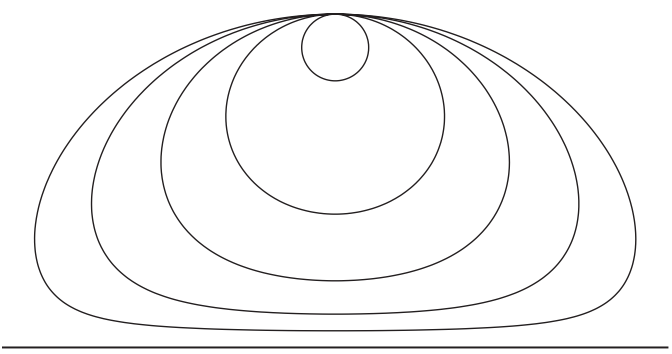

FIGURE 7. Vortex patch shapes for $d=0.05,0.1,0.2,0.4,0.8$ satisfying the Kutta condition and $a=1$. The aspect ratio of the plot is one.

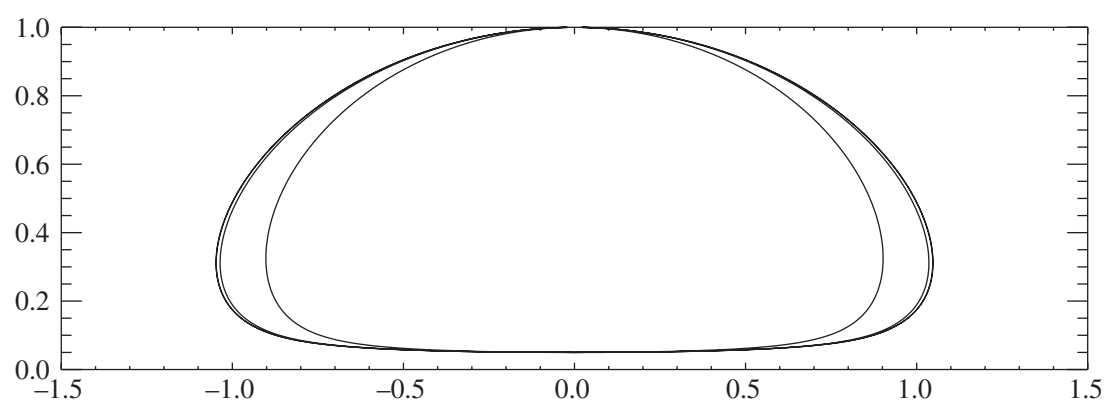

FIGURE 8. Vortex patch shapes of increasing area for $a=1,2,4, \infty$ satisfying the Kutta condition and $d=0.05$.

4.2. Kutta condition Figure 7 shows the shapes of equilibrium vortex patches above a plate of length $a=1$. As in the zero circulation case, as $d$ increases, the patch becomes more circular, but for given $d$ the Kutta condition patches have a larger aspect ratio than their zero circulation counterparts.

Figure 8 shows the effect of increasing the plate length $a$ on the shape of the vortex. As $a$ increases, the area of the patch increases. Compared to the case when there is zero circulation about the plate, the approach to the infinite wall shape is more rapid: for $a \geq 2$ the infinitely long wall case provides a good approximation for the shape of the patch.

Figure 9 shows properties of the equilibria for $d=0.05$ as a function of $a$ (choosing $a=0.5,0.65,0.8,1,1.25,1.5,2,3,4,5,6,7,8,9,10)$. Again agreement is good even when the patch is clearly noncircular. Finally the lift exhibits a maximum magnitude when the plate half-length is close to one, and then decays slowly to zero as $a$ increases. This is consistent with the point vortex result (2.10) which shows $L \sim a^{-1}$ as $a \rightarrow \infty$.

\section{Time-dependent behaviour}

The robustness of the computed steady solutions is tested by using them as initial conditions in a time-dependent contour dynamics simulation. The algorithm is 

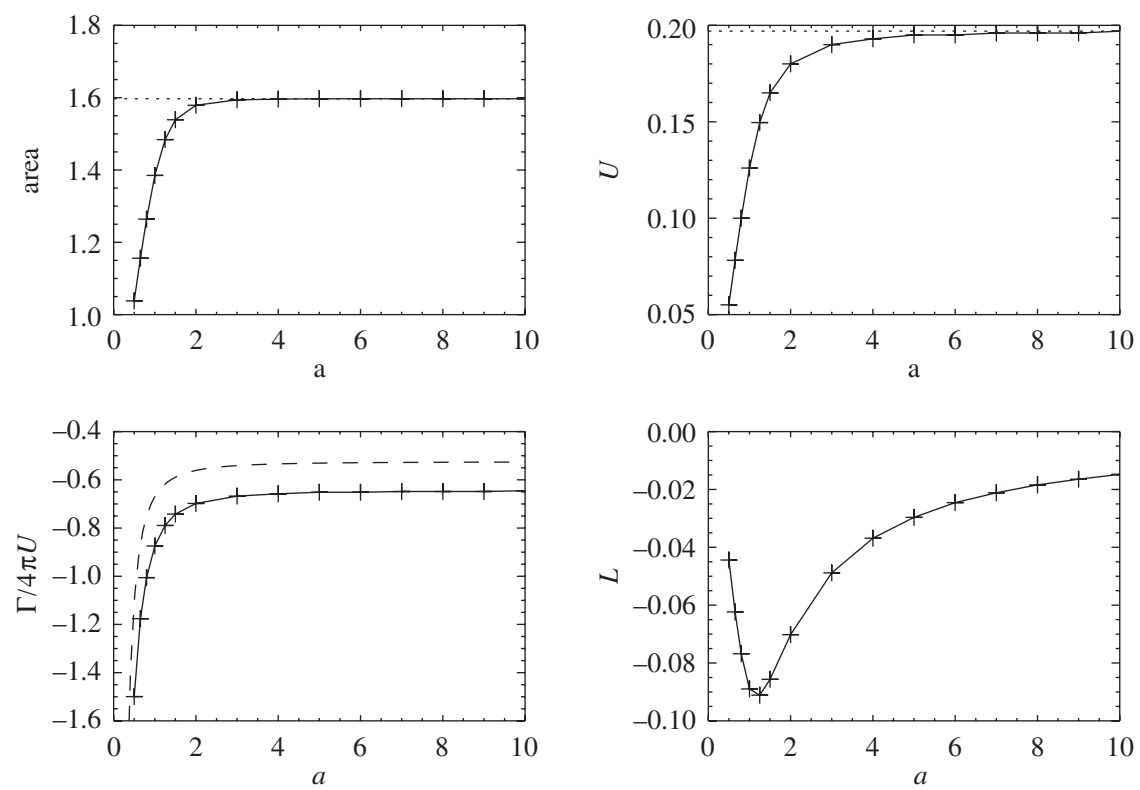

FIGURE 9. Equilibria properties of patch area, $U, \Gamma / 4 \pi U$ and lift $L$ for $d=0.05$ as a function of $a$ when the Kutta condition is satisfied. In the top two plots the dotted line represents $U$ and patch area near an infinitely long wall. The approach to these asymptotic values is more rapid than in the zero circulation case. In the third plot the dashed line represents the value of $\Gamma / 4 \pi U$ for a point vortex (2.9).

essentially the same as outlined in Section 3 for calculating the velocities at the nodes on the patch boundary due to the patch itself and the presence of the plate. Once these velocities are known the nodes are advected using a fourth-order Runge-Kutta method [3]. Since inevitably there are small numerical errors, unstable equilibria are expected to diverge from their initial configuration whereas stable equilibria are expected to remain close to their initial state. Although this test is not a proof of the stability properties of an equilibrium solution it is an extremely good indication of these properties.

Equilibria with $a=1$ and $d=0.05$ were tested for times up to $t=500$ (where, recall, time has been nondimensionalized using $|\omega|^{-1}$ ) and using a timestep of 0.1 . In the zero circulation case the equilibrium was found to be stable. That is, the patch boundary at $t=500$ was found to coincide virtually identically with its initial configuration. On the other hand, the equilibrium corresponding to the Kutta condition initially drifted very slowly from its initial location (see Figure 10), that is, at $t=400$ the patch had moved slightly to the right. Thereafter the patch evolved much more rapidly and by $t=500$ had moved significantly from its equilibrium position. It then rapidly moves away from the plate as it is advected by the mean flow.

These time-dependent experiments lead to the conclusion that the equilibria with zero circulation about the plate are stable whereas those in which the Kutta condition 


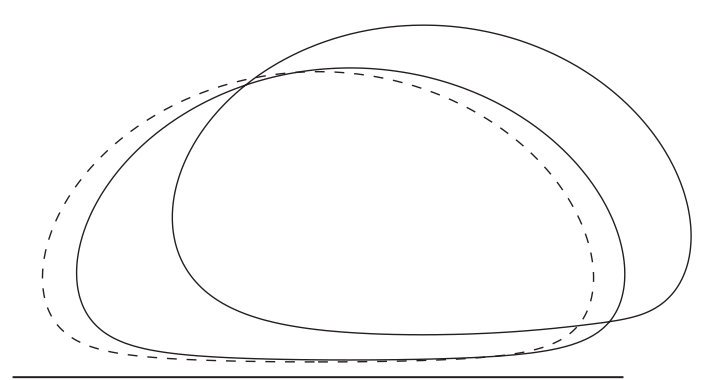

FigurE 10. Time-dependent evolution of a vortex patch for Kutta flow and $d=0.05$ and $a=1$. The initial $(t=0)$ equilibrium patch shape is shown (dashed curve) and snapshots of the patch are shown at $t=400$ and $t=500$.

is satisfied are unstable (although they are able to survive coherently near the plate for significant times). This behaviour is consistent with Section 2 where it was shown that the point vortex equilibria correspond to either elliptic (stable) or hyperbolic (unstable) points of the Hamiltonian.

\section{Conclusions}

Symmetric vortex patches above a flat plate in equilibrium with uniform flow parallel to the plate have been found numerically. The configuration allows for an arbitrary circulation around the plate and two families of vortex equilibria have been found corresponding to either zero circulation about the plate or when the Kutta condition is satisfied at the plate ends. Both families can be thought of as being desingularized point vortex equilibria. Given the distance of the outer edge of the plate, each family depends on two parameters, namely the plate length and the distance of the innermost edge of the patch from the plate. The shape of the patch, lift on the plate and stream velocity are then determined. For given $a$ and $d$ this determination appears to be unique: starting the numerical routine from an initial shape other than a circle (that is, ellipses of varying aspect ratio) failed to find any further solutions.

Comparison of numerical results for patches with the explicit point vortex results derived in Section 2 suggests that results for the point vortex equilibria with circulation equal to that of the patch predict well the properties of the equilibria even when the patch is far from circular in shape. This suggests that the point vortex model is appropriate for use in, say, aerodynamic modelling. Of course, finite area patch results are still necessary to find the correct area of the patch and hence the circulation of the equivalent point vortex.

Time-dependent simulations suggest that the equilibria are stable in the zero circulation case but unstable in the Kutta case, although they are able to persist for significant times in this latter case. These stability properties are consistent with the behaviour of point vortices as deduced from the nature of the equilibrium points of their respective Hamiltonians. 


\section{References}

[1] G. K. Batchelor, "Steady laminar flow with closed streamlines at large Reynolds numbers", J. Fluid Mech. 1 (1956) 177-190.

[2] G. Deem and N. J. Zabusky, "Stationary "V-states", interactions, recurrence and breakings", Phys. Rev. Lett. 40 (1978) 859-862.

[3] D. G. Dritschel, "Contour surgery: a topological reconnection scheme for extended integrations using contour dynamics", J. Comput. Phys. 77 (1988) 240-266.

[4] A. Elcrat, B. Fornberg, M. Horn and K. Miller, "Some steady vortex flows past a circular cylinder", J. Fluid Mech. 409 (2000) 13-27.

[5] A. Elcrat, B. Fornberg and K. Miller, "Stability of vortices in equilibrium with a circular cylinder", J. Fluid Mech. 544 (2005) 53-68.

[6] G. C. Hocking, "Steady Prandtl-Batchelor flows past a circular cylinder", ANZIAM J. 48 (2006) $165-177$.

[7] E. R. Johnson and N. R. McDonald, "The motion of a vortex near two circular cylinders", Proc. Roy. Soc. A 460 (2004) 939-954.

[8] E. R. Johnson and N. R. McDonald, "Vortices near barriers with multiple gaps", J. Fluid Mech. 531 (2005) 335-358.

[9] E. R. Johnson and N. R. McDonald, "Steady vortical flow around a finite plate", Q. J. Mech. Appl. Math. 60 (2007) 65-72.

[10] L. M. Milne-Thompson, Theoretical hydrodynamics, 2nd edn (Macmillan, London, 1949).

[11] R. T. Pierrehumbert, "A family of steady, translating vortex pairs with distributed vorticity", J. Fluid Mech. 99 (1980) 129-144.

[12] E. J. Routh, "Some applications of conjugate functions", Proc. Lond. Math. Soc. 12 (1984) 73-89.

[13] P. G. Saffman, Vortex dynamics (Cambridge University Press, Cambridge, 1992).

[14] P. G. Saffman and J. S. Sheffield, "Flow over a wing with an attached free vortex", Stud. Appl. Math. 57 (1977) 107-117.

[15] P. G. Saffman and S. Tanveer, "Prandtl-Batchelor flow past a flat plate with a forward facing flap", J. Fluid Mech. 143 (1984) 351-365.

[16] H. M. Wu, E. A. Overman and N. J. Zabusky, "Steady state solutions of the Euler equations in two-dimensions. Rotating and translating V-states with limiting cases", J. Comput. Phys. 53 (1984) 42-71.

[17] L. Zannetti, "Vortex equilibrium in flows past bluff bodies", J. Fluid Mech. 562 (2006) 151-171. 